\title{
Traffic optimization in IOT networks
}

\author{
Hassan OUAHI ${ }^{1}{ }^{a}$,Abdenbi MAZOUL ${ }^{1}$ \\ ${ }^{1}$ Laboratory Computing Systems \& Vision LabSiv, \\ University Ibn Zohr of Agadir, \\ Faculty of Science, BP 28/S, Agadir 80000, \\ MOROCCO \\ h.ouahi@uiz.ac.ma,a.mazoul@uiz.ac.ma
}

Keywords: IoT,IPv4,IPv6,Routing,RFID.

\begin{abstract}
Since the 2000 s, the idea that the Internet could be used for machine-to-machine communication and process automation has emerged. Together with the development of electronic objects capable of communicating with IP protocols, this idea led to the concept of the Internet of Things (IoT Internet of Things). Nowadays, the evolution of networks is very intense. New networks are appearing, "all-optical" solutions in the heart of networks, "wireless" solutions to facilitate access to users or to implant sensors / actors in places difficult to access, or finally the Internet of communicating objects. In this evolution, new scientific issues and challenges are emerging. Some of the problems are related to the optimization of generated traffic as well as the improvement of the performance of tools and techniques. Often, these optimization problems lead to models using graphs. The analysis of optimization problems in networks is an important activity. IOT networks create more data traffic that is difficult to control within the operator's network. The method given in this paper will help network operators optimize their networks and assist developers in improving hardware and software interactions to reduce data traffic in the network.
\end{abstract}

a Chttps://orcid.org/0000-0000-0000-0000

b Dhttps://orcid.org/0000-0000-0000-0000 


\section{INTRODUCTION}

In recent years, Internet use has reached 3 billion people on the basis of surveys conducted by United Nations agency[1]. The Internet of Things ( IoT) is known to be the interconnection between the internet and real world objects and people that extends the idea of traditional human-to - machine interconnection to the machine.

Interconnection in which things [2] are singularly identified by providing them with unique addresses. The sensing capacity is usually not provided by traditional internet, so only the computers are interconnected.

But the IoT does have sensing capability as the sensors [3] are installed in the IoT devices along with Radio Frequency Identification ( RFID) tags [4] and actuators, allowing contact between the devices [5]. Kevin Ashton [6] first used the word IoT in a presentation in 1998, the Internet-based information service architecture represented a growing globe.

There are many fields where IoT can be applied or introduced, including smart houses, manufacturing, infrastructure, military, traffic management and many more.

Embedded with sensors, actuators and RFID tags are the real world stuff and objects whether they are human, animal, vehicle, refrigerator, ATM or something else in the real world [7].

The sensors sense the environment and collect the data, and actuators perform specified actions for a particular case.

The data collected was sent to the Gateways, then to the Internet [8]. If a customer asks for a specific one Service whether it be business or healthcare, their data is relevant Provides real time for the consumer as shown in Figure 1.

In IoT, applications consist of intrinsic as an actuator, sensors, Radio Frequency IDentification ( RFID), and communication interfaces including Global Positioning Systems (GPS), infrared,

Bluetooth, Wireless LANs [9].

The IoT devices are linked to each other using an inter-transfer protocol to transmit the information.

This link lets us collect more data from more industries.

IoT is a framework that connects the system sensors and the data networks.

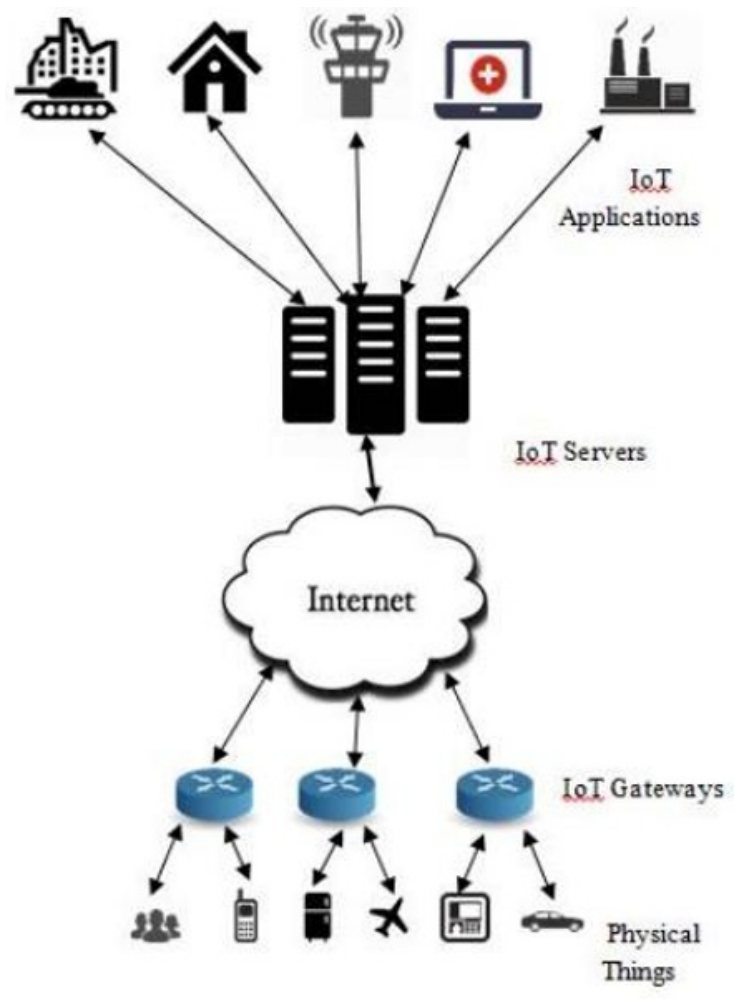

Fig. 1: A Typical IoT Architecture

Routing plays a crucial role in systems operating IoT. Routing is a very challenging factor that exists in IoT due to its intrinsic properties.

Often routing protocol called as routing policy, which specifies how routing devices communicate with each other in the network, circulating control information that selects the best routes among multiple routes between any two nodes;

Data can be exchanged from a source node by nearest neighbors in the routing protocol, and enters the sink node.

It determines the best route between the source and the destination node based on algorithms in routing it.

The routing protocol must be configured to efficiently transmit data over the network with minimum energy consumption, as the battery capacity is limited for IoT nodes [10] and multiple routes from one node to another must also be established to minimize the delay in the event of route or node failure.

In this article, we propose a object type routing protocol, which maintains paths using object type from one node to another and also provides Internet connectivity to the nodes in the network and thus maximize the life of the network and reduce traffic. 
The rest of this paper is organized as follows: In section 2, we discuss the related works relevant to our proposed work.

Section 3 provides our proposed protocol and a detail operation. In section 4, we evaluate the performance of the proposed system. Finally, we conclude to our work in section 5 .

\section{RELATED WORK}

Routing algorithms are used to decide the routes the data should follow, and some of the properties such as the routes between nodes should be chosen such that the data reaches its destination in the best possible way.

Based on certain criteria the best way can be specified according to the application requirements. For example, finding and using the route with the lowest end-to - end delay, or the highest throughput, is one very important metric, while another might be using the route with minimal hop distance or the best quality of link.

Therefore, in order to enable the nodes in the network to transmit data to each other, the routing protocol is required to provide the route between them that it determined. Since the IoT network is very large, which means a huge number of things, extreme network complexity and devices are resource constrained; The routing may be affected by frequent changes in topology and irregular connectivity that impose severe routing challenges [11]. Internet of Things ( IoT) is a wireless network, with nodes roaming freely. With insufficient battery power the network output is affected by node and path failures [12]. Multipath routing approach must therefore be considered when designing the routes that will provide alternate route to the nodes if failure occurs and thus minimize the overall delay. Various multitrack approach routing protocols have been developed. To provide a multipath routing solution for the network Le, Q. Et al.[13] supported for modified RPL (IPV6 Low Power Routing Protocol) techniques And Lossy Network protocol that extends simple RPL protocol. The authors outlined major limitations of the RPL routing protocol with respect to the lack of multi-path routing based on the construction and maintenance of DODAGs (Destination Oriented Directed Acyclic Graph) to root data from sensor nodes over a single path.

In order to overcome this limitation, the authors proposed three multi-path schemes based on the classical RPL: Energy Load Balancing-ELB, Fast
Local Repair-FLR and the combination of these two ELB-FLR, then the schemes are combined into an IoT modified IPv6 communication stack.

First scheme, ELB resolves unbalanced load in RPL; it proposes a new set of objective functions to calculate rank based on both hop-count and residual energy. Second scheme, FLR is planned to minimize the number of local repairs in order to do this FLR uses a new term sibling by having more route continuity to use in urgent situations. Third scheme ELB-FLR, a combination of two previous methods combines ELB's objective function and load balancing, faster local repair, and FLR's loop detection / evasion into RPL.

Tian and Y. Et al.[14] has proposed an improved AOMDV routing protocol (Ad hoc On Demand Multipath Distance Vector) for IoT route design. The aim of this updated ad-hoc on-demand multipath distance vector is to find node-disjunction and link-disjunction routes and also to connect nodes and a secure internet connection.

The protocol will dynamically select the secure Internet transmission path by updating the Internet Connecting Table periodically.

Due to the requirement of additional Internet Connecting Table with routing table, the routing overhead is more in this Protocol.

Qiu, T.Et al [15] proposed an Efficient MultiPath Self-Organization Strategy on the Internet of Things to improve the tolerance of faults and to coordinate the efficiency of the GEAR wireless network protocol based on geographical location information in the IoT network and to achieve better energy conservation and distribution results. Thus the authors suggested SMG, Self-organized Multipath GEAR, a modern multi-path routing organizing protocol based on the traditional sensor network GEAR regional routing protocol.

\section{PROPOSED SHEME}

In our approach we try to find a solution to reduce network traffic and do object type routing in IOT networks.

For this reason the structure of the IPv4 figure 2 [16] and IPv6 figure 3 [17] datagram's was used. 


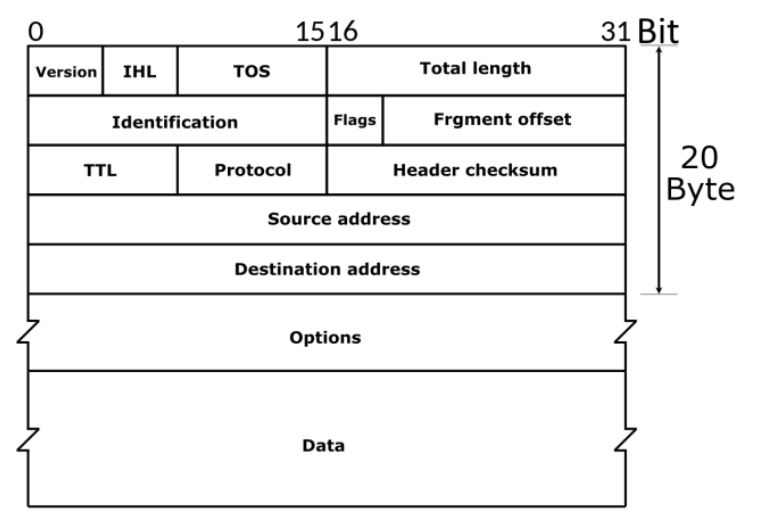

Fig. 2: IPv4 Structure

\begin{tabular}{|c|c|c|c|}
\hline & 1 & & \\
\hline Version & Traffic class & \multicolumn{2}{|c|}{ Flow label } \\
\hline \multicolumn{2}{|r|}{ Payload length } & Next header & Hop limit \\
\hline \multicolumn{4}{|c|}{ Source address } \\
\hline \multicolumn{4}{|c|}{ Destination address } \\
\hline \multicolumn{4}{|c|}{ Extensions } \\
\hline
\end{tabular}

Fig. 3: IPv6 Structure

In the IPv4 options field or IPv6 extensions field we put an information that allows us to determine the type of object to which the packet is destined, for example we take 2 bytes for this field, we represent the lamps by 000000000001 , we represent the air conditioners by 000000000010 , the windows by $000000000011, \ldots$; and that way if we want to send a message to a set of lamps (20 for example) with the same order, we send a single packet instead of 20, so we reduce the bandwidth consumption, and the memory occupation of the transit routers figure 4 .

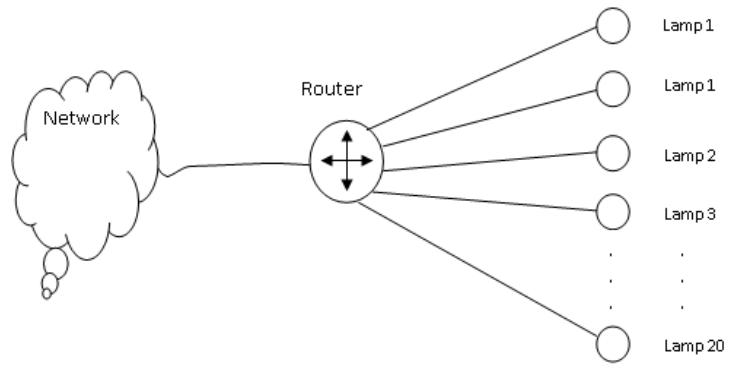

Fig. 4:IoT Network object type

\section{RESULTS}

Taking for example an industry that contains 100 windows, 200 air conditioners, 300 lamps and 300 motors, we want to know the state of these different objects. Are the windows open, air conditioners started, lights on and engines started?

If we use simple routing we have to send $100+200+300+300=900$ packets, but if we use our object type routing approach, we have to send 4 packets because we have four object types and that way we will avoid sending 896 packets.

If we generalize this to all industries and public institutions (high school, school...) we will reduce a significant number of packets and thus optimize the backbone bandwidth, as well as the consumption of network equipment resources.

\section{CONCLUSIONS}

We have proposed a routing approach that is based on object type. Traditional routing is in relation to the proposal creates several packets when addressing objects of the same type. The results of our approach show that we perform routing that reduces bandwidth consumption in IoT networks and generates less traffic.

Analysis of the proposed protocol for relatively more Internet connection nodes and the runtime mobility of the nodes can be carried out considered to improve the proposed Protocol in the future, the proposed study can be applied in more practical setting and its success can be checked.

\section{REFERENCES}

[1] Money INTERNET, Available at: http://time.com/money/3896219/internet-users-worldwide/ accessed May 2015. 
[2] L. Atzori, A. Iera and G. Morabito, "The Internet of Things: A survey," Comput. Networks, vol. 54, no. 15, pp. 2787-2805, 2010.

[3] J. Gubbi, R. Buyya, and S. Marusic, "Internet of Things (IoT ): A Vision , Architectural Elements , and Future Directions," vol. 29, no. 1, pp. 1-19, 2013.

[4] D. Giusto, A. Iera, G. Morabito, L. Atzori (Eds.), "The Internet of Things," Springer, ISBN: 987-14419-1673-0, 2010.

[5] A., Al-Fuqaha, M., Guizani, M., Mohammadi, M., Aledhari, and M., Ayyash, "Internet of things: A survey on enabling technologies, protocols, and applications", Communications Surveys \& Tutorials, IEEE, vol. 17, no. 4, pp. 2347-2376, 2015.

[6] M. Wu, T. J. Lu, F. Y. Ling, J. Sun, and H. Y. Du, "Research on the architecture of Internet of Things," in proc. Of 3rd Int. Conf. Adv. Comput. Theory Eng. Proc., vol. 5, pp. 484-487, 2010.

[7] I. Mashal, O. Alsaryrah, T. Y. Chung, C. Z. Yang, W. H. Kuo, and D. P. Agrawal, "Choices for interaction with things on Internet and underlying issues," Ad Hoc Networks, vol. 28, no. January, pp. 68-90, 2015.

[8] S.K. Malik, M. Dave, S. K. Dhurandher, I. Woungang, and L. Barolli, "An ant-based QoSaware routing protocol for heterogeneous wireless sensor networks," Soft Computing, pp.1-12, 2016.

[9] K Zhao, L Ge. A survey on the internet of things security. InComputational Intelligence and Security (CIS). 2013 ; 663667.

[10] A., Dhumane, R., Prasad, and J., Prasad, "Routing Issues in Internet of Things: A Survey," Proceedings of the International Multi-Conference of Engineers and Computer Scientists, Vol. 1, pp. 16-18, 2016.

11] S. Kumar, M. Dave and S. Dahiya. "QoS Enabled Probabilistic Routing for Heterogeneous Wireless Sensor Networks." International Journal of Computer Network and Information Security, vol. 5, no. 4, pp. 31-39, 2013.

12 A. Arya, A. Malik, and S. Kumar. "A routing protocol for detecting holes in wireless sensor networks with multiple sinks." In Proceedings of the Third International Symposium on Women in Computing and Informatics, pp. 103-108. ACM, 2015.

13 Q. Le, T. Ngo-Quynh, and T. Magedanz, "RPLbased multipath Routing Protocols for Internet of Things on Wireless Sensor Networks," 2014 Int. Conf. Adv. Technol. Commun. (ATC 2014), pp. 424-429, 2014.

14 Y. Tian and R. Hou, "An improved AOMDV routing protocol for internet of things," 2010 Int. Conf. Comput. Intell. Softw. Eng. CiSE 2010, pp. $0-3,2010$.

15 T., Qiu, W., Sun, Y., Bai, and Y., Zhou, "An Efficient Multi-Path Self-Organizing Strategy in Internet of Things," Wireless personal communications, vol. 73, no. 4, pp. 1613-1629, 2013.

16 https://www.ietf.org/rfc/rfc791.txt

17 https://tools.ietf.org/html/rfc2460 